\title{
REDUCING RADIO BANDWIDTH LOAD IN NANOLOC-BASED WIRELESS NETWORKS THROUGH SELECTING APPROPRIATE SUBSET OF BASE STATIONS FOR RANGING
}

\author{
Alexander S. Galov ${ }^{1)}$, Alex P. Moschevikin ${ }^{1,2)}$, Alexei V. Soloviev ${ }^{2)}$ \\ ${ }^{1)}$ RTL-Service ltd., Petrozavodsk, Russian Federation \\ ${ }^{2)}$ Petrozavodsk State University, \\ 31 Lenin Str., IT-Park of PetrSU, 185910, Petrozavodsk, Russian Federation \\ gas@lab127.ru, alexmou@rtlservice.com, avsolov@lab127.ru
}

\begin{abstract}
In wireless sensor networks based on nanoLOCTM standard and using server-centric control, the overall performance of radio segment and location accuracy depends amongst other on the efficiency of the location engine. The efficiency may be increased by selecting an appropriate subset of base stations for ranging. This paper describes the experiments dealing with this problem, and discusses the ways of saving radio bandwidth.
\end{abstract}

Keywords: real time location systems, wireless sensors networks, ranging, accuracy, nanoLOCTM, time-of-flight, round trip time, geometric dilution of precision.

\section{INTRODUCTION ${ }^{1}$}

Modern local positioning systems or RTLS (realtime location systems) are based on various wireless technologies: WiFi, ZigBee, nanoLOC ${ }^{\mathrm{TM}}$ etc. The main goal of such systems is to determine local position (coordinates) of a wireless mobile device (tag) that may be carried by a human or mounted on a machine, equipment etc. RTLS is a very important instrument to solve the problems of logistics, staff management and security.

The typical RTLS includes a set of base stations (BS) with known geographical coordinates and a set of wireless tags to be located. There are two main approaches for location calculation in wireless sensors networks: based on time-of-flight (ToF) and received signal strength (RSS) [1]. Information acquisition, data processing and implementation of business logics are performed by a server.

As a rule wireless tags have very short periods of radio activity to reduce power consumption and increase the time between recharges. They transmit special blink frame indicating active state. Base stations (anchors) receiving this radio frame retransmit its copies to a server, which issues commands to base stations to start the ranging

\footnotetext{
${ }^{1}$ The research described in this publication was financed by RTL-Service ltd. and supported by Petrozavodsk State University and the Ministry for economical development of Republic of Karelia (Russian Federation).
}

procedure.

The performance of a typical local positioning system based on wireless sensors network depends amongst many other things - on two factors: available traffic load and the number of calculated locations per second. These parameters are mutually dependant and, in addition, depend on the efficiency of location engine of the system.

In an ideal case, it is sufficient to collect only three ranging results to calculate the position of the tag. But in practice in order to achieve stability and reliability in wireless communications, the density of base stations should be higher than the required minimum. So the server should not receive only three copies of an "I am alive" frame, but probably up to 10.

To save bandwidth it is reasonable to minimize the number of base stations which carry out distance measurement to the mobile tag. The main problem concerns the selection of the appropriate base stations from the set.

Many methods help to solve this problem. Geometric dilution of precision (GDOP) is a well known parameter for the analysis of satellites configuration in the GPS [2-4]. Nowadays GDOP is widely used as a criterion for choosing the right geometric configuration of base stations in wireless sensors networks [5-9]. It can be applied, for example, for evaluating positioning accuracy for different positioning algorithms [5] or for improving 
performance [6]. In the majority of positioning systems GDOP is the most promising criterion for selecting an appropriate subset of base stations for ranging to increase the positioning accuracy. Nevertheless, the paper shows that this algorithm may be improved by adding a few technical criteria.

Section 2 demonstrates that the problem of selecting an appropriate subset of base stations is very important for accuracy and performance of RTLS. Section 3 describes the basic algorithm of applying GDOP and the possibilities of its improvement. The simplified principle of location calculation in the systems based on ToF approach is described in Section 4. In Section 5 we present the ongoing project of development of TalkLoc ${ }^{\mathrm{TM}}$ technology, which implements the proposed algorithm. Section 6 compares the effectiveness of various modifications of GDOP algorithm through experimental results. Conclusions are drawn in Section 7.

\section{PROBLEM OF SELECTING AN APPROPRIATE SUBSET OF BS}

The impact of satellites disposition on positioning accuracy is well studied for the global positioning systems [10]. The same problem exists in local positioning systems based on wireless sensors network.

Due to the errors caused by non-line-of-sight (NLOS) signal propagation the distance between a mobile unit and a base station measured by ToF method is always above or equals the real distance [11]. That is why circles corresponding to the distances measured from several base stations do not intersect in one point and form a certain location area (see figure 1).

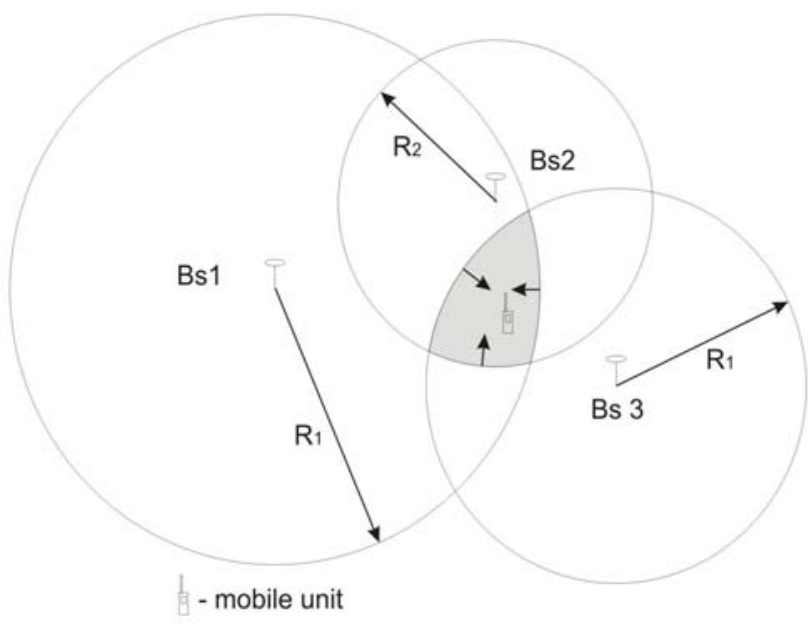

Fig 1 - Typical location area

(Bs1, Bs2, Bs3 - base stations)

If there are no errors in measurements except NLOS error a mobile unit is inside this area with
$100 \%$ probability. With the use of a specific algorithm (it can be MLS, KF or other algorithms $[12,13,14])$ it is possible to clarify the mobile unit position inside the location area.

Geometric configuration of base stations can strongly influence the shape of a location area and thus location accuracy. Let's consider two sets of three base stations (A and B in figure 2) with different positions of BSs as to the mobile unit position. In the first set (A) a mobile unit is homogeneously surrounded by base stations. In the second set (B) base stations are placed on the one side from a mobile unit. Circle radii from base stations $\mathrm{BS} 1, \mathrm{BS} 2$, and $\mathrm{BS} 3$ in set (A) are equal to circle radii for corresponding base stations BS1, BS2, and BS3 from the set (B). The distances between the mobile unit and the nearest points of the circles are equal. These distances are characterized by NLOS component. Although NLOS component is the same for the two sets, the location area in case (A) is much smaller than in case (B).

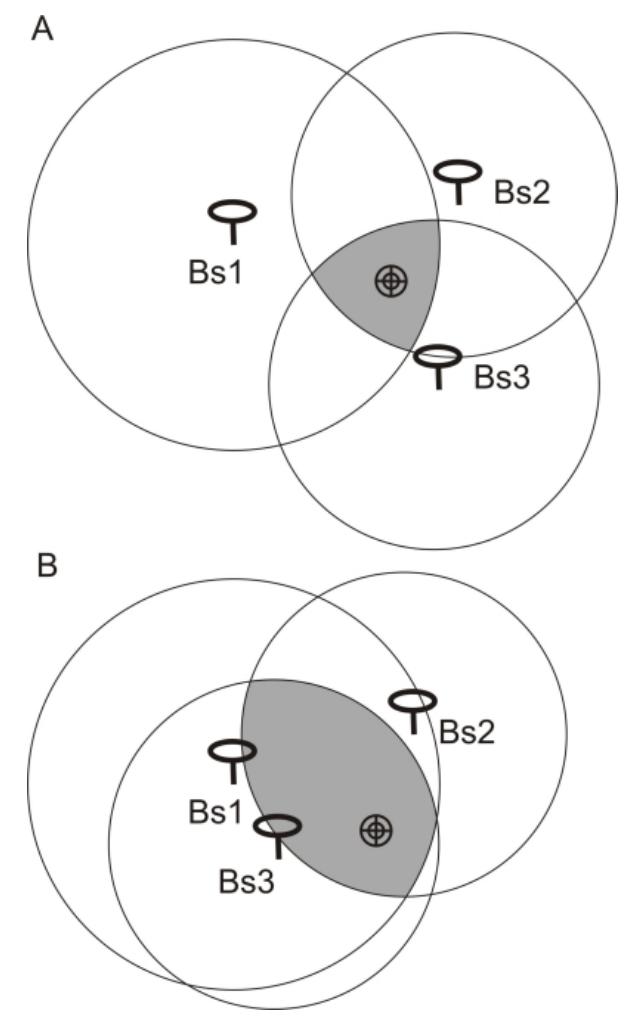

Fig. 2 - The impact of base station placement on the shape of location area

The optimal geometric configuration of BSs is important even in the case when the estimation of the measurements error is known.

Two characteristic pictures (A) and (B) of BSs' geometric configuration with known estimation of measurements error are presented in figure 3 . 


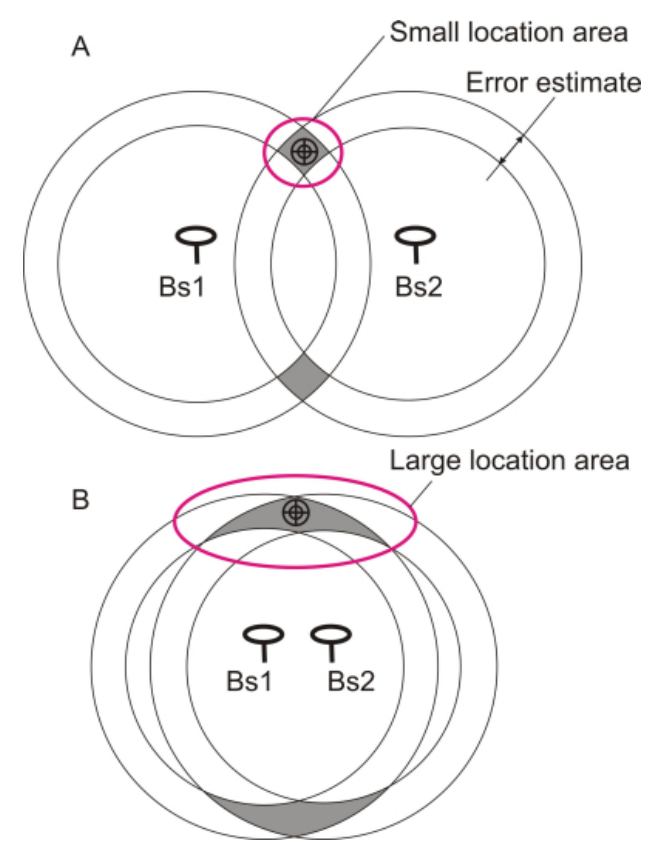

Fig. 3 - The impact of BSs' geometric configuration on the shape of location area (known error estimation)

Rings around the base stations BS1 and BS2 correspond to the most probable distances of a tag (taking into account error estimation). In case (A) the base stations are far apart from a mobile node, giving relatively small areas of possible mobile unit location. In case $(\mathrm{B})$ the errors in measurements are equal to the errors in case (A), but the base stations are closer to each other. As a result the areas of possible mobile unit location in case (B) are larger than the areas in case (A).

\section{GEOMETRIC DILUTION OF PRECISION}

One of the basic parameters used in GPS for the analysis of satellites disposition is GDOP [2-4, 15]. This parameter can be applied for the analysis of BSs' geometric configuration in the considered wireless sensors network. For the 2D case GDOP is calculated in three steps.

1. First it is necessary to calculate matrix $A$ as

$$
A=\left[\begin{array}{cc}
\frac{\left(X a_{1}-x\right)}{R_{1}} & \frac{\left(Y a_{1}-y\right)}{R_{1}} \\
\frac{\left(X a_{2}-x\right)}{R_{2}} & \frac{\left(Y a_{2}-y\right)}{R_{2}} \\
\cdots & \cdots \\
\frac{\left(X a_{n}-x\right)}{R_{n}} & \frac{\left(Y a_{n}-y\right)}{R_{n}}
\end{array}\right],
$$

where $\left(X a_{1}, Y a_{1}\right), \ldots,\left(X a_{\mathrm{n}}, Y a_{\mathrm{n}}\right)$ are the coordinates of the base stations, $(x ; y)$ are the coordinates of the mobile unit, $R_{i}$ is the distance measured between a base station with coordinates $\left(X a_{i}, Y a_{i}\right)$ and a mobile unit.

2. Matrix $G$ is calculated as

$$
G=\left(A^{T} A\right)^{-1},
$$

$G$ can be written as

$$
G=\left[\begin{array}{ll}
g_{11} & g_{12} \\
g_{21} & g_{22}
\end{array}\right] .
$$

3. GDOP value is calculated as

$$
\text { GDOP }=\left(g_{11}+g_{22}\right)^{2}
$$

GDOP value can be used as a criterion for the selection of the appropriate subset of base stations to carry out ranging. If it is necessary to choose several base stations from the given set for the distance measurements, the subset with the lowest GDOP should be chosen.

For the use of GDOP in real-time analysis of BSs' optimal geometric configuration it is necessary to know the approximate position of a mobile unit beforehand. There are several ways to estimate the preliminary position of a mobile unit. It depends on the applied positioning algorithm.

In the case of applying Kalman Filter [16] it is possible to use prognosis estimation of a target position. In the case of applying MLS and if the ranging is frequent enough it is possible to use previous position for GDOP calculation. If no prior information is available it is possible to evaluate location by RSS measurements.

\section{APPLIED ALGORITHM FOR LOCATION CALCULATION}

To analyze the impact of geometric configuration of BSs on location accuracy we used MLS algorithm to calculate locations of a mobile unit. It is rather simple algorithm, but easily demonstrates the proposed solution since ranging was performed by ToF method.

The coordinates $\hat{X}, \hat{Y}$ of a wireless tag's position were calculated as

$$
\left(\begin{array}{c}
\hat{X} \\
\hat{Y}
\end{array}\right)=\left(H^{T} H^{-1}\right) H^{T} Z
$$

where

$$
H=\left[\begin{array}{cc}
2 X a_{1}-2 X a_{2} & 2 Y a_{1}-2 Y a_{2} \\
2 X a_{1}-2 X a_{3} & 2 Y a_{1}-2 Y a_{3} \\
\ldots & \ldots \\
2 X a_{1}-2 X a_{n} & 2 Y a_{1}-2 Y a_{n}
\end{array}\right]
$$




$$
Z=\left[\begin{array}{c}
R_{2}^{2}-R_{1}^{2}+X a_{1}^{2}-X a_{2}^{2}+Y a_{1}^{2}-Y a_{2}^{2} \\
R_{3}^{2}-R_{1}^{2}+X a_{1}^{2}-X a_{3}^{2}+Y a_{1}^{2}-Y a_{3}^{2} \\
\cdots \\
R_{n}^{2}-R_{1}^{2}+X a_{1}^{2}-X a_{n}^{2}+Y a_{1}^{2}-Y a_{n}^{2}
\end{array}\right]
$$

If information on statistics of measurement errors is available it is possible to use symmetric weight matrix $Q$.

$$
Q=\left\|q_{i j}\right\|
$$

With the use of weight matrix the coordinates of a mobile unit are calculated as

$$
\left(\begin{array}{l}
\hat{X} \\
\hat{Y}
\end{array}\right)=\left(H^{T} Q H\right)^{-1} H^{T} Q z .
$$

Matrix $Q$ is the inverse of the noise covariance matrix

$$
Q=\left(\begin{array}{ccccc}
\sigma_{1}^{-2} & 0 & 0 & \ldots & 0 \\
0 & \sigma_{2}^{-2} & & \ldots & 0 \\
0 & 0 & \sigma_{3}^{-2} & \ldots & 0 \\
\cdots & \ldots & \ldots & \ldots & \ldots \\
0 & 0 & 0 & 0 & \sigma_{N}^{-2}
\end{array}\right),(9)
$$

where $\sigma_{\mathrm{k}}$ is standard deviation of measurements error for $k$-th base station (measurements are considered to be independent). These deviations were found during preliminary experiments.

The proposed algorithm for location calculation was tested using TalkLoc ${ }^{\mathrm{TM}}$ technology.

\section{TALKLOC ${ }^{\mathrm{TM}}$ TECHNOLOGY}

$\mathrm{TalkLoc}^{\mathrm{TM}}$ is a wireless technology characterized by low power consumption, ranging and voice communication [17]. It is based on nanoLOC ${ }^{\mathrm{TM}}$ (IEEE 802.15.4a) radio standard, developed by Nanotron Technologies GmbH. Technical characteristics of nanoLOC ${ }^{\mathrm{TM}}$ chips are presented in table 1 .

\section{Table 1. NanoLOCTM key features [18]}

\begin{tabular}{|l|l|}
\hline Modulation technique & Chirp Spread Spectrum \\
\hline Radio band & $2.4-2.48 \mathrm{GHz}$ ISM \\
\hline Ranging accuracy & $2 \mathrm{~m}$ indoors / 1 m outdoors \\
\hline Output power & Up to $20 \mathrm{dBm}$ \\
\hline Data rates & $250 \mathrm{kbps}$ to $1 \mathrm{Mbps}$ \\
\hline Ranging method & $\begin{array}{l}\text { time-of-flight, round trip } \\
\text { time }\end{array}$ \\
\hline
\end{tabular}

In nanoLOC ${ }^{\mathrm{TM}}$ ranging is based on time-of-flight (ToF) method. According to the round trip time (RTT) scheme two radio nodes exchange radio frames and register the times of frames transmission, processing and reception. One of the nodes collects the time data and then performs the calculations of the distance between them. The simplified scheme is presented in figure 4 .

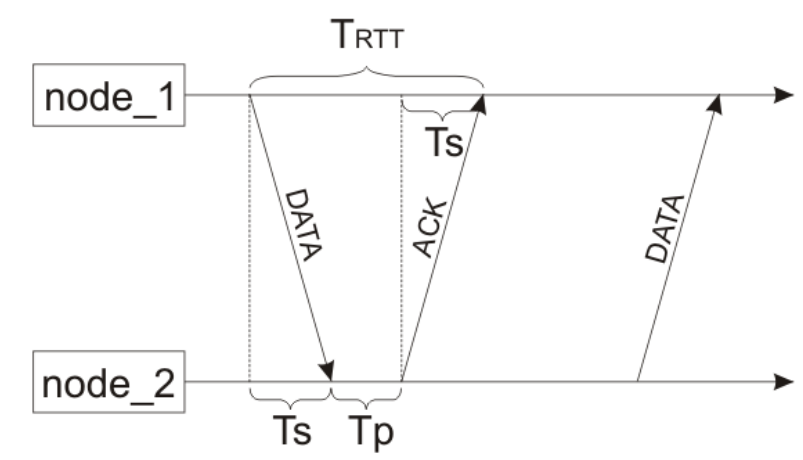

Fig. 4 - Round trip time ranging scheme

To initiate distance measurement node_1 transmits DATA frame. Time Ts corresponds to the signal propagation between node_1 and the node_ 2 . Hardware of node 2 receives this frame and processes it (time $\mathrm{Tp}$ ) and then sends the acknowledgement frame ACK. In the next DATA frame node_2 sends the value of $\mathrm{Tp}$ to node 1 . Node 1 registers the moment of reception of ACK frame, measures $\mathrm{T}_{\mathrm{RTT}}$ time and then calculates the distance $\mathrm{D}$ according to the following formula:

$$
D=\left(T_{R T T}-T p\right) * c / 2
$$

where $c$ - speed of light.

NanoLOCTM chip also provides measurements of received signal strength (RSS) for every frame reception. This information can also be used for position calculation [19-21].

In TalkLocTM systems a radio coverage zone is created by a set of base stations connected to the backbone. For example it may be constructed with the use of optical or copper Fast or Gigabit Ethernet channels. A special dedicated server controls the performance of the wired and wireless subsystems. Besides control functions, it processes and stores the obtained data. In TalkLocTM applications it is used for communications with software clients and phones as well.

As it was mentioned above the larger part of the cycle of the wireless tag corresponds to the power saving mode, tags do not receive radio signals during this period of time. When the specified interval elapses the wireless tag wakes up and broadcasts "I am alive" packet. During a short period of time the device stays in the active state and awaits commands from the server. All the base stations that receive "I am alive" packet re-transmit its copies to the server. These copies are taken into account when the server initiates series of distance measurement commands (commands to base stations to carry out next ranging cycle between them and a mobile tag).

Obviously, location measurement commands can not be sent simultaneously, so the server forms a queue of commands. The next command from the queue is transmitted upon receiving a reply on the 
previous command. In wireless sensors networks such reply can be lost or not generated at all. In such cases several commands may be not performed and the location measurement cycle for a certain tag will not be finished correctly. It is very important to plan the queue of location measurement commands in the way that obtains the larger number of reliable results. And if timeout occurred the acquired results of ranging would be enough to estimate the location of the wireless tag adequately.

\subsection{ADDITIONAL CRITERIA FOR BASE STATION SELECTION}

When selecting base stations for a location measurement cycle a few technical considerations should be taken into account as well.

Unless a wireless tag and a base station are located in the range of reliable communication an attempt to perform ranging may fail.

On the other hand, the fact that the server has not received a copy of "I am alive" frame from a certain base station at the instance of initiation of the location measurement cycle does not mean that the base station is not in the reception range of the wireless tag and the ranging is not possible.

For example, this situation may be explained by packets delays. We tested some systems where base stations were interconnected by means of wired and wireless networks introducing essential delays. Delays up to several hundreds of milliseconds occurred in the QLAN segment, delays up to tens of milliseconds occurred in the WiFi segment. Taking into account this fact the algorithm considers the copies of both the last and preceding "I am alive" frame. Thereby, the following values may be proposed as the additional criteria for base station selection:

- the received signal strength;

- the number of failed measurements of location between the base station and the wireless tag (the history of failures should not be very long, because it quickly loses relevance for moving tag);

- the moment when the last copy of "I am alive" frame was obtained through the base station (whether it is the last frame or the preceding frame).

The second and the third criteria are very important for $2.4 \mathrm{GHz}$ radio networks with high probability of interference.

Also an additional criterion of priority (or base station "importance") may be introduced. A base station that unambiguously determines the territory of location (a floor) or is equipped with higherefficiency antenna may be considered as a higherpriority base station. Any organizational reasons may be considered as priority as well.

\section{RESULTS}

The experiments to analyze the impact of BSs' geometric configuration on positioning accuracy were conducted in the area of hotel resorts where the real-time location system based on TalkLoc ${ }^{\mathrm{TM}}$ technology was installed. The size of the area was $620 \times 270$ meters. Solid dots on the scheme in figure 5 correspond to the positions of the base stations.

The territory included many buildings (cottages) especially in zone A. Therefore in this region there were no so many places where base stations and a mobile unit were on the line of sight. Another part of the hotel was the beach area (zone B in figure 5). The line-of-sight conditions were perfect for the major part of the area. The main building (5 floors) corresponded to zone $\mathrm{C}$ in figure 5 . In order to achieve better accuracy in the case of large percentage of NLOS measurements made indoors the density of base stations was the highest in zone C.

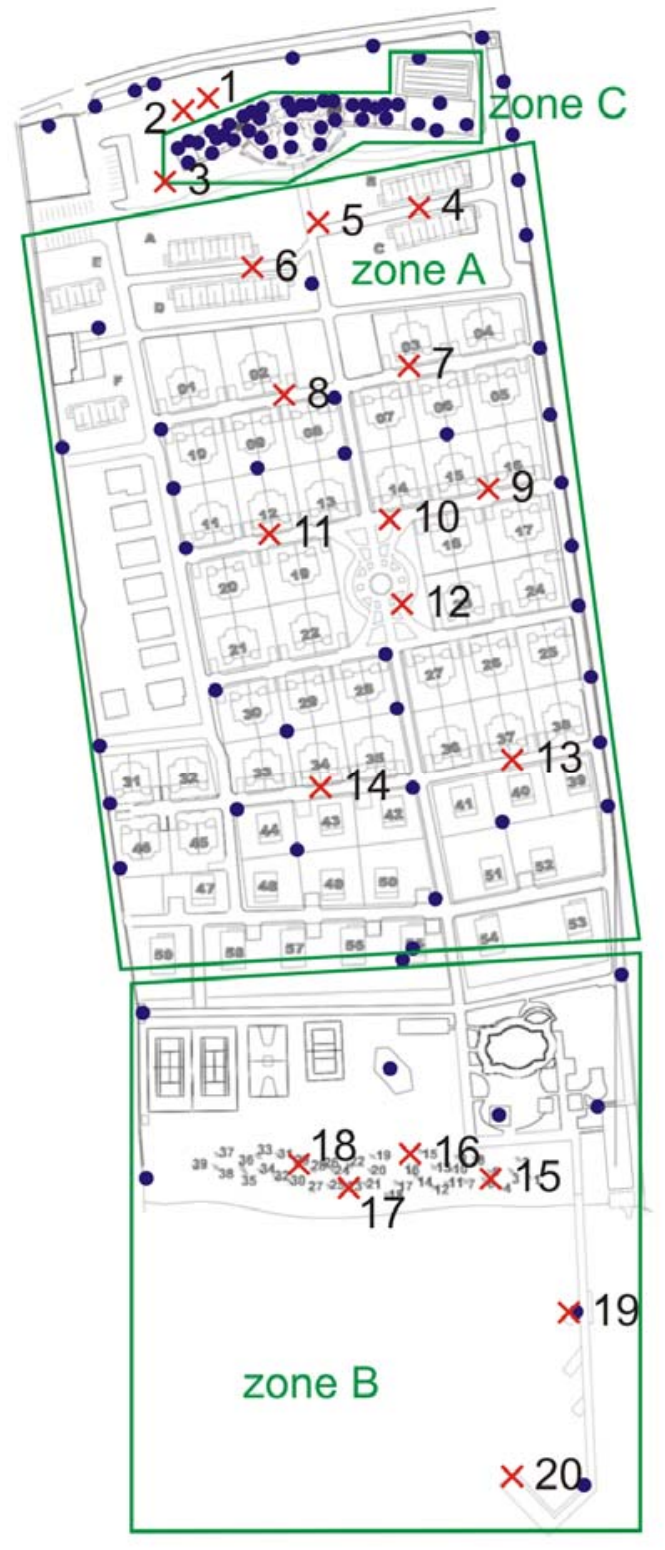

Fig. 5 - Hotel scheme 
The experiments were carried out in all zones A, $\mathrm{B}$ and C. In all 20 experimental points (marked with crosses with the corresponding numbers) data were collected within 5 minutes. The interval between rangings was 1 second. Only one mobile unit was used to avoid excessive load of base stations.

The experiments were conducted in daytime therefore the intensity of measurement noise caused by moving obstacles at different periods of time was approximately equal.

In all experimental points at least 8 base stations were available for subsequent ranging: all of them transferred "I am alive" copies to the server. So for every point $C_{8}^{4}=70$ combinations of base stations were available for range measurements. To analyze the impact of BSs' geometric configuration on location accuracy, the mobile unit position was calculated for each combination of 4 base stations from 8 available in each selected point (figure 5). If more than 8 base stations received a packet from the tag, only 8 closest base stations were taken into account.

In every point for a certain subset of the base stations both GDOP value and location of the mobile unit were calculated. These data were used to calculate error cumulative distribution function and determine $90 \%$ error border.

For all available subsets GDOP parameter varied from 1.1 up to 11.5. Positioning error (R90\%) varied from 2.3 meters for the best subset of the base stations to 13.1 meters for the worst subset.

Calculated GDOP values, quantity of the subsets with the specified GDOP range and corresponding positioning errors intervals are summarized in table 2 .

Table 2. GDOP intervals, quantity of the subsets with the specified GDOP range and corresponding positioning errors (R90\%).

\begin{tabular}{|c|c|c|}
\hline $\begin{array}{c}\text { GDOP value } \\
\text { ranges }\end{array}$ & $\begin{array}{c}\text { Quantity of } \\
\text { subsets, \% }\end{array}$ & $\begin{array}{c}\text { Positioning } \\
\text { errors (R90\%), } \\
\text { m }\end{array}$ \\
\hline $1-2$ & $47 \%$ & $2.3-4.3$ \\
\hline $2-3$ & $21 \%$ & $3.8-5.6$ \\
\hline $3-4$ & $16 \%$ & $4.7-6.1$ \\
\hline $4-5$ & $9 \%$ & $5.5-7.2$ \\
\hline $5-6$ & $4 \%$ & $5.8-11.1$ \\
\hline$>6$ & $3 \%$ & $8.1-13.1$ \\
\hline
\end{tabular}

The example of the subset that gives the best accuracy is shown in figure 6 .

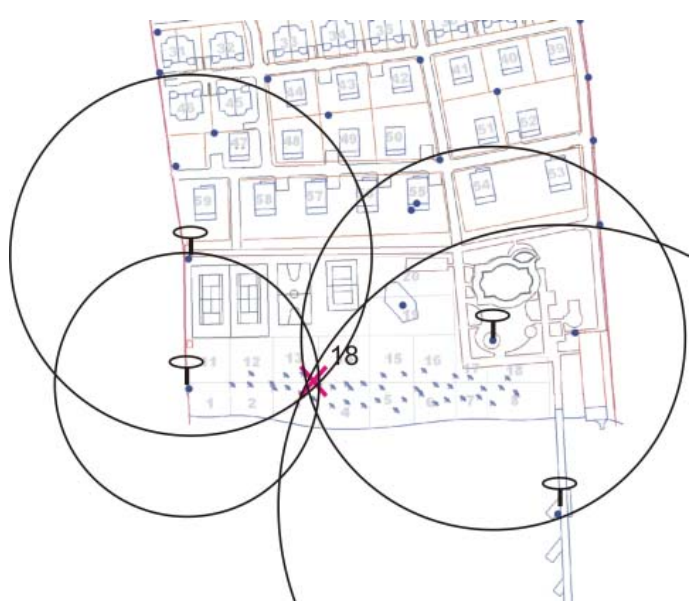

Fig. 6 - The best anchor subset corresponding to the lowest positioning error

As predicted the area with the best accuracy was observed in zone B of the hotel area. This zone is characterized by the absence of obstacles. Therefore the NLOS error is minimal for this zone.

The worst subset for the beach area is shown in figure 7 .

As we can see for the worst case (lowest accuracy) all four base stations were grouped to the north-east from the experimental point, while in the case of the best accuracy all base stations homogeneously surrounded the experimental point.

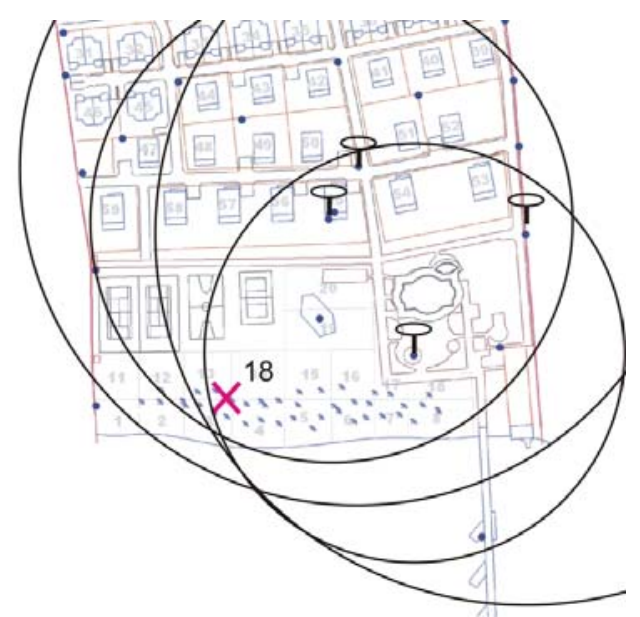

Fig. 7 - Example of the worst anchor subset

Analyzing all the experiments the majority of subsets have GDOP value less than 2 (see table 2). The location accuracy for these configurations is in the range of $2.3-4.3$ meters. It is not clear what subset should be chosen from this set to perform ranging. To choose the best subset we used signal strength as an additional criterion for base stations selection.

The combined online positioning algorithm with the use of GDOP and signal strength consists of several steps.

1) On the basis of preliminary location estimation examine all possible subsets of the base stations and calculate GDOP values. 
2) Divide the available GDOP values into a set of intervals (1-2), (2-3), etc.

3) Select the subsets with the best GDOP range (e.g. (1-2) if such subsets exist).

4) Use the subset with the highest RSS values within the selected GDOP range.

\subsection{POSITIONING OF A MOBILE UNIT WITH THE USE OF THE PROPOSED ALGORITHM}

The experiments of mobile unit positioning were conducted for three different methods of subsets selection.

The first method corresponds to the selection of a base stations subset based only on RSS values. From the available base stations set the given number of base stations which have the highest RSS values was selected for distance measurements.

The second method corresponds to GDOP criterion: the number of base stations which have the lowest GDOP values was selected for measurements.

And the third method corresponds to the combination of GDOP and RSS criteria. This method was described above.

The results of positioning accuracy for these criteria for a subset of 4 anchors from 8 available are shown in figure 8 .

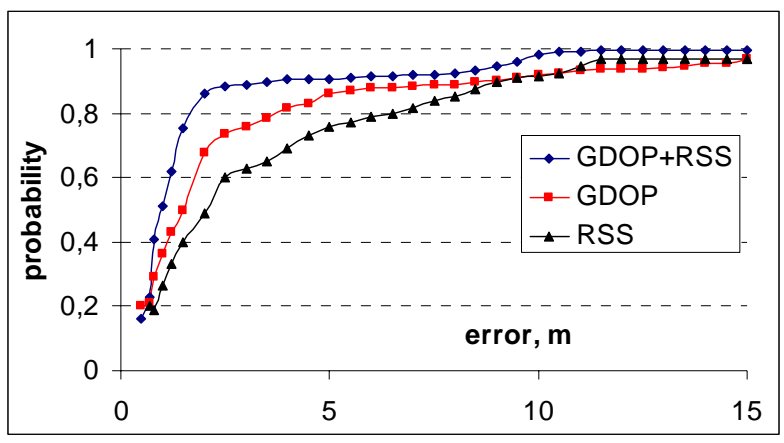

Fig. 8 - Error cumulative distribution function representing position calculation efficiency using subset of 4 BS for different selection criteria

As we can see in figure 8 the best location accuracy was achieved when the combination of two factors GDOP and RSS was used for base stations selection. The GDOP criterion represents better localization performance than RSS. One of the possible reasons is that in the case of GDOP criterion the subset is chosen so that it compensates measurements errors. However additional information that signal strength provides can be used for selection of more adequate measurements. A multipath signal often has less RSS value than a line-of-sight signal. That is why the combination of GDOP and RSS criteria represents the best localization performance.

\subsection{VARYING THE NUMBER OF BASE STATIONS}

Several tests on varying the number of base stations available for measurements have been performed. The experimental conditions were the same as in previous experiments. The data on location accuracy were collected for all experimental points depicted in figure 5 .

Two techniques of base stations selection were used. The first technique uses random selection of several anchors from the given set, and the second is based on combination of GDOP and RSS criteria. The quantity of selected anchors in a subset varied from 4 to 8 .

Figure 9 shows the result of the experiment with the use of the first technique. For the random base station selection the location inaccuracy $(90 \%$ threshold) for the subset of 4 from 8 base stations was more than 15 meters. The best location accuracy was 3.1 meters for 8 base stations. The difference between the best and the worst cases is above 12 meters.

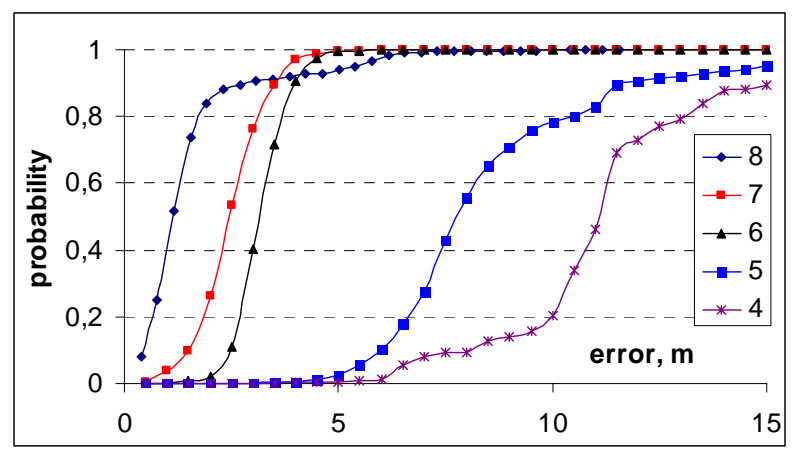

Fig. 9 - Location accuracy for varying number of base stations in subset used for location calculation (random BS selection)

Figure 10 shows the results of the experiments for the proposed base stations selection algorithm (combination of GDOP and RSS criteria).

The difference between location accuracy for $90 \%$ threshold for the best and the worst cases is less than 1 meter. The best accuracy was achieved with a subset of 6 base stations. The accuracy in this case was better than 2.6 meters.

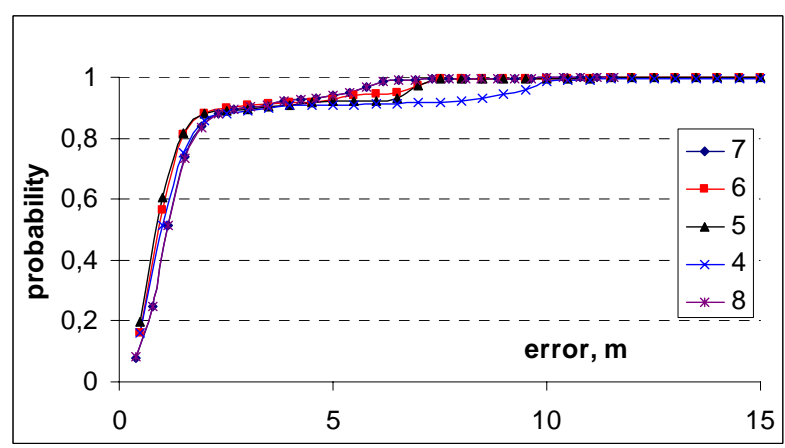

Fig. 10 - Location accuracy for varying number of base stations in subset used for location calculation (GDOP+RSS BS selection) 
It should be mentioned that the location accuracy for 6 anchors was better than for all 8 anchors. Possibly the measurements from 2 excessive base stations might introduce additional error due to NLOS signal propagation.

\section{CONCLUSION}

In this paper the role of the impact of BSs' geometric configuration on location accuracy in local positioning systems was analyzed.

The proposed algorithms may be used both for increasing location accuracy and for radio bandwidth saving.

The proper subset from the set of available base stations seems to be a more important factor for location accuracy than the number of base stations.

The conducted experiments proved that a correct choice of geometric configuration of BSs can increase positioning accuracy in local positioning systems by several times and, thus, save radio bandwidth.

The authors are ready to share raw ranging data with researchers who wish to test their algorithms and to compare their effectiveness.

\section{ACKNOWLEDGEMENTS}

The authors would like to thank Edward Levin and Oksana Dobrynina for useful censorious remarks.

\section{REFERENCES}

[1] M. Vossiek, L. Wiebking, P. Gulden, J. Wieghardt, C. Hoffmann, P. Heide, Wireless Local Positioning, Microwave Magazine, (4) 4 (2003), pp. 77-86.

[2] Richard B. Langley, Dilution of Precision, GPS World, May 1999, pp. 52-59.

[3] E. D. Kaplan, C. J. Hegarty, Understanding GPS: Principles and Applications, 2nd ed., Artech House Press, Boston, 2006.

[4] N. Levanon, Lowest GDOP in 2-D scenarios, Proc. IEEE Radar, Sonar and Navigation, (147) (2000), pp. 149-153.

[5] Chien-Sheng Chen, Jium-Ming Lin, WenHsiung Liu, Ching-Lung Chi, Dilution of position calculation for MS location improvement in wireless communication systems, Journal of Networks, (6) 10 (2011).

[6] Lin-Chih Chu, Po-Hsuan Tseng, and Kai-Ten Feng, GDOP-Assisted location estimation algorithms in wireless location systems, Proc. of IEEE "GLOBECOM" 2008.

[7] Israel Martin-Escalona, Francisco BarceloArroyo, Impact of geometry on the accuracy of the passive TDOA algorithm, Proc. of IEEE 19th International Symposium on Personal Indoor and Mobile Radio Communications, 2008.

[8] N. Patwari, J. Ash et al, Locating the nodes: cooperative localization in wireless sensor networks, IEEE Signal Processing Magazine, (22) 4 (2005), pp. 54-69.

[9] Damien B. Jourdan, Nicholas Roy, Optimal Sensor Placement for Agent Localization, Proc. of the IEEE/ION Position, Location and Navigation Symposium (PLANS 2006), San Diego, April 2006.

[10] J. J. Spilker, Satellite constellation and geometric dilution of precision, Global Positioning System: Theory and Applications, vol. 1. Washington, D.C., 1996, pp. 177-208.

[11] A. Gogolev, B. Ekimov, K. Ekimov, A. Moschevikin, A. Fedorov, I. Tsykunov, Accuracy of distance measuring using nanoLOC technology, Wireless Technologies, (12) 3 (2008), pp. 48-51. (in Russian)

[12] Rainer Mautz, Overview of current indoor positioning systems, Geodezija ir kartografija / Geodesy and Cartography, (35) 1 (2009), pp. 18-22.

[13] C. Rohrig, M. Muller, Localization of sensor nodes in a wireless sensor network using the nanoLOC TRX transceiver, Proc. of Vehicular Technology Conference, April 2009, IEEE 69th Barcelona, pp. 1-5.

[14] N. Patwari, J. N. Ash, S. Kyperountas, A. O. Hero, R. L. Moses, N. S. Correal, Locating the nodes: cooperative localization in wireless sensor networks, IEEE Signal Processing Magazine, (22) 4. (2005), pp. 5469.

[15] Richard B. Langley, Dilution of Precision, GPS World, May 1999, pp. 52-59.

[16] Peter S. Maybeck, The Kalman filter: an introduction to concepts", Autonomous Robot Vehicles, I.J. Cox, G. T.Wilfong (eds), Springer-Verlag, 1990.

[17] www.talkloc.com

[18] Real Time Location Systems (RTLS), Nanotron Technologies GmbH, Berlin, Germany, White paper NA-06-0248-0391-1.02, Apr. 2007

[19] Gayathri Chandrasekaran et al., Empirical evaluation of the limits on localization using signal strength, IEEE SECON 2009, June 2009.

[20] Eiman Elnahrawy, Xiaoyan Li, and Richard P. Martin, The limits of localization using signal strength: a comparative study, IEEE SECON, Santa Clara, California, USA, October 2004, pp. 406-414.

[21] A. Galov, A. Moschevikin, R. Voronov, Combination of RSS localization and ToF 
ranging for increasing positioning accuracy indoors, Proc. of ITST-2011, St-Petersburg, Russia, August 2011, pp. 299-304.

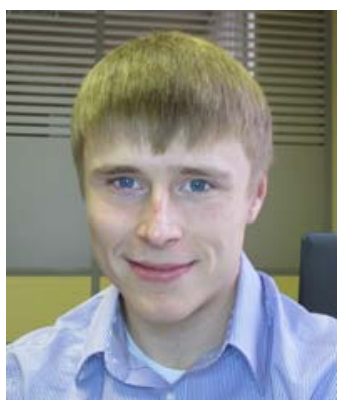

Alexander S. Galov, PhD student at Faculty of Physical Engineering of Petrozavodsk State University, principal engineer in small innovative enterprise RTL-Service Itd., Russian Federation.

The focus of his research is location algorithms in wireless sensors networks.

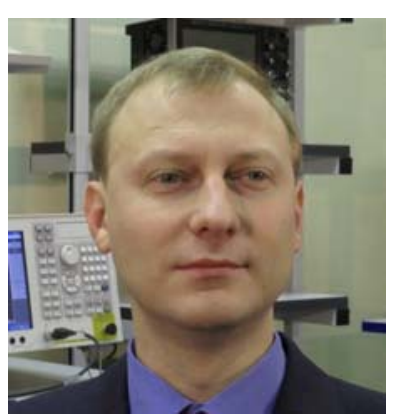

Alex P. Moschevikin, PhD, associate professor at Faculty of Physical Engineering of Petrozavodsk State University, chief science officer of small innovative enterprise RTL-Service Itd., Russian Federation.

Dr. Moschevikin is the leader of the research team (25 developers) which carried out several projects on wireless sensors networks, automation, computer nets, Internet technologies and distributed remote software and hardware development.

The products of RTL-Service Itd. won several prizes at International exhibitions.

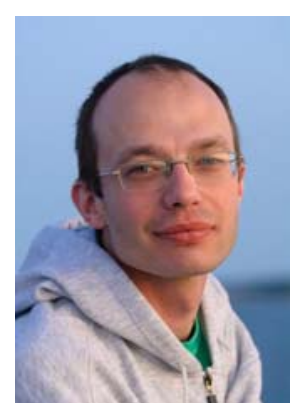

Alexei V. Soloviev, PhD, associate professor at Faculty of Physical Engineering of Petrozavodsk State University.

Dr. Soloviev is one of the leading experts of Petrozavodsk State University in the field of telecommunications and network technologies. He is the author of several tutorials on automation and network operating systems. 